\title{
ON REARRANGEMENTS OF SERIES
}

H. M. SENGUPTA

In an interesting paper published in the Bulletin of the American Mathematical Society, R. P. Agnew ${ }^{1}$ considers some questions on rearrangement of conditionally convergent series.

He considers the metric space $E$ in which a point $x$ is a permutation $\left(x_{1}, x_{2}, x_{3}, \cdots\right)$ of the positive integers and in which the distance $\rho(x, y)$ between two points $x \equiv\left(x_{1}, x_{2}, x_{3}, \cdots\right)$ and $y \equiv\left(y_{1}, y_{2}, y_{3}, \cdots\right)$ of $E$ is given by the Fréchet formula

$$
\rho(x, y)=\sum_{n=1}^{\infty} \frac{1}{2^{n}} \frac{\left|x_{n}-y_{n}\right|}{1+\left|x_{n}-y_{n}\right|} .
$$

Agnew proves that the space $E$ is of the second category at each of its points.

He also considers the following problem: Let $C_{1}+C_{2}+C_{3}+\cdots$ be a conditionally convergent series of real terms. Denote $C_{n}$ by $C(n)$. To each $x \in E$ there corresponds a rearrangement $\sum_{n=1}^{\infty} C\left(x_{n}\right)$ of the series $\sum C(n)$ or $\sum C_{n}$ and also to each rearrangement of the series corresponds a point $x \in E$. Thus the ways in which the series may be rearranged form a set which has the potency of $E$. It is well known that $x \in E$ exists for which $\sum_{n} C\left(x_{n}\right)$ converges to any preassigned number, diverges to $+\infty$ or to $-\infty$, or oscillates with prescribed upper and lower limits. The set $A$ of $x \in E$ for which $\sum_{n=1}^{\infty} C\left(x_{n}\right)$ converges is therefore a proper subset of $E$. Professor Agnew considers the nature of the set $A$ and proves that it is of the first category so that the complementary set $E-A$ is of the second category. In point of fact he proves more than that. He shows that the set of points $x \in E$ for which $\sum_{n=1}^{\infty} C\left(x_{n}\right)$ has unilaterally bounded partial sums is of the first category. His first theorem runs as follows:

For each $x \in E$, except those belonging to a set of the first category,

$$
\liminf _{N \rightarrow \infty} \sum_{n=1}^{N} C\left(x_{n}\right)=-\infty, \quad \limsup _{N \rightarrow \infty} \sum_{n=1}^{N} C\left(x_{n}\right)=+\infty .
$$

It is possible to add something more to the above in regard to the nature of the set $A$. In fact we can prove the following theorem:

Received by the editors November 28, 1947 and, in revised form, August 16, 1948.

1 Ralph Palmer Agnew, On rearrangement of series, Bull. Amer. Math. Soc. vol. 46 (1940) pp. 797-799. 
Theorem 1. For each $x \in E$, except those of a set $B\left(F_{0}\right.$ in $\left.E\right)$ that is an outer-limiting set of sets closed in $E$, we have

$$
\liminf _{N \rightarrow \infty} \sum_{n=1}^{N} C\left(x_{n}\right)=-\infty, \quad \limsup _{N \rightarrow \infty} \sum_{n=1}^{N} C\left(x_{n}\right)=+\infty .
$$

Combining the above with the result given in Agnew's paper, we may assert that the set of points $x$ for which

$$
\liminf _{N \rightarrow \infty} \sum_{n=1}^{N} C\left(x_{n}\right)=-\infty \quad \text { and } \quad \limsup _{N \rightarrow \infty} \sum_{n=1}^{N} C\left(x_{n}\right)=+\infty
$$

is the inner limiting set of a sequence of everywhere dense sets open in $E$.

Proof. We require the following ideas for the proof of our theorem. Consider the series $\sum_{n=1}^{\infty} C\left(x_{n}\right)$. Let $s_{1}, s_{2}, s_{3}, \cdots, s_{N}, \cdots$, where $s_{N}=\sum_{n=1}^{N} C\left(x_{n}\right)$, be the partial sums.

Let us denote the maximum of the numbers $\left(s_{1}, s_{2}, \cdots, s_{N}\right)$ by $U_{N}$. Then $s_{1}, U_{2}, U_{3}, \cdots$ is a monotone nondecreasing sequence of real numbers, and $\lim _{N \rightarrow \infty} U_{N}$ exists either as a finite number or as $+\infty$. We write $\lim _{N \rightarrow \infty} U_{N}$ as $\lim _{N \rightarrow \infty}$ upper bound $\sum_{n=1}^{N} C\left(x_{n}\right)$.

In a similar manner if $u_{N}$ denotes min $\left(s_{1}, s_{2}, \cdots, s_{N}\right)$, then $s_{1}, u_{2}, u_{3}, \cdots$ is a monotone nonincreasing sequence of real numbers and $\lim _{N \rightarrow \infty} u_{N}$ exists either as a finite number or as $-\infty$. We denote $\lim _{N \rightarrow \infty} U_{N}$ by the symbol $\lim _{N \rightarrow \infty}$ lower bound $\sum_{m=1}^{N} C\left(x_{n}\right)$.

Now, let $h>0$ be large at pleasure. We define $B_{h}$ to be the set of points of $E$ for which $\lim _{N \rightarrow \infty}$ upper bound $\sum_{n=1}^{N} C\left(x_{n}\right) \leqq h$.

Now let $\xi_{1}, \xi_{2}, \xi_{3}, \cdots$ be a convergent sequence of points of $E$ all belonging to $B_{h}$. Let their limit point $\xi$ also belong to $E$. We shall prove that $\xi$ also belongs to $B_{h}$.

Let $\xi_{n} \equiv\left(x_{1}^{(n)}, x_{2}^{(n)}, x_{3}^{(n)}, \cdots\right)$ and $\xi \equiv\left(x_{1}, x_{2}, x_{3}, \cdots\right)$. The number complexes $\left(x_{1}^{(n)}, x_{2}^{(n)}, x_{3}^{(n)}, \cdots\right)$ for $n=1,2,3, \cdots$ and also the complex $\left(x_{1}, x_{2}, x_{3}, \cdots\right)$ are different permutations of the positive integers.

If now $\xi$ does not belong to $B_{k}$ we must have

$$
\lim _{N \rightarrow \infty} \text { upper bound } \sum_{n=1}^{N} C\left(x_{r}\right)>h \text {. }
$$

So, for sufficiently large $N$, say $N \geqq M^{\prime}$ where $M^{\prime}$ is some positive integer depending on $\xi$ and $h$, we must have $U_{N}>h$.

In particular, therefore, $U_{M^{\prime}}>h$. But $U_{M^{\prime}}$ is the maximum of $\left(s_{1}, s_{2}, s_{3}, \cdots, s_{M^{\prime}}\right)$, so there is a positive integer $M\left(1 \leqq M \leqq M^{\prime}\right)$ such that $s_{M}>h$, that is, there is a positive integer $M$ for which

$$
S_{M}=C\left(x_{1}\right)+C\left(x_{2}\right)+\cdots+C\left(x_{M}\right)>h .
$$


We may now take a sphere of sufficiently small radius to ensure that the first $M$ elements of all points of $E$ that lie in the interior of the sphere are identical with those of $\xi$ in value and in order. So for all these points the corresponding rearranged series are'such that the $M$ th partial sum of each of them equals $S_{M}=C\left(x_{1}\right)+C\left(x_{2}\right)+\ldots$ $+C\left(x_{M}\right)$ and so exceeds $h$ in value.

Now, since $\lim \xi_{n}=\xi$, for all sufficiently large $n$, say $n \geqq P$ where $P$ is a suitable positive integer, $\xi_{n}$ lies within the sphere referred to above. So

$$
\sum_{r=1}^{M} C\left(x_{r}^{(n)}\right)=C\left(x_{1}\right)+C\left(x_{2}\right)+\cdots+C\left(x_{M}\right)>h
$$

for $n=P, P+1, P+2, \cdots$. Therefore

$$
\varliminf_{N \rightarrow \infty} \text { upper bound } \sum_{r=1}^{N} C\left(x_{r}^{(n)}\right)>h \quad(n=P, P+1, \cdots),
$$

that is, $\xi_{p}, \xi_{p+1}, \xi_{p+2}, \cdots$ all belong to $E-B_{h}$ which is contrary to the hypothesis. So $\xi$ belongs to $B_{h}$, that is, the set $B_{h}$ is closed in $E$.

In a like manner, we may prove that if $B_{-\lambda}$ stands for the set of points of $E$ for which

$$
\underset{N \rightarrow \infty}{\lim \text { lower bound }} \sum_{r=1}^{N} C\left(x_{r}\right) \geqq-h
$$

and if $\left\{\zeta_{1}, \zeta_{2}, \zeta_{2}, \cdots\right\}$ is a convergent sequence of points of $E$ all belonging to $B_{-k}$ with $\zeta$ as the limit which itself belongs to $E$, the $\zeta \in B_{-\mu}$. So, $B_{-h}$ is closed in $E$.

It follows therefore that for any positive integer $n$, the set $B_{n}+B_{-n}$ is closed in $E$, that is, the set of points of $E$, for which

$$
\lim _{N \rightarrow \infty} \text { upper bound } \sum_{r=1}^{N} C\left(x_{r}\right) \leqq n
$$

or

$$
\lim _{N \rightarrow \infty} \text { lower bound } \sum_{r=1}^{N} C\left(x_{r}\right) \geqq-n
$$

is closed in $E$.

The set $B$ referred to above is, therefore, given by

$$
B=\sum_{n=1}^{\infty}\left(B_{n}+B_{-n}\right) \text {. }
$$

So, $B$ is a set $F_{\sigma}$ in $E$, that is, the outer limiting set of a sequence of sets closed in $E$. 
It follows that the set of points $x \in E$ for which

$$
\liminf _{N \rightarrow \infty} \sum_{n=1}^{N} C\left(x_{n}\right)=-\infty \text { and } \limsup _{N \rightarrow \infty} \sum_{n=1}^{N} C\left(x_{n}\right)=+\infty
$$

is $G_{\delta}$ in $E$.

We next propose to derive some simple properties about the power of $E$.

ThEOREM 2. The set $E$ has the power $c$ of the continuum.

Proof. Let $p$ be the power of the set $E$. Let us associate the point $x=\left(x_{1}, x_{2}, x_{3}, \cdots\right)$ of the set $E$ to the irrational number $\alpha$ which is the value of the simple continued fraction

$$
\frac{1}{x_{1}}+\frac{1}{x_{2}}+\frac{1}{x_{3}}+\cdots \text {. }
$$

Thus to each point $x \in E$ there corresponds an irrational number $\alpha$ in $0<\alpha<1$ and to two distinct points $x$ and $y$ of $E$ correspond two distinct irrational numbers in the above range. Thus the set $E$ is equivalent to a proper subset of the real numbers. Therefore

$$
p \leqq C \text {. }
$$

On the other hand, to any real number $\beta(0<\beta<1)$ there corresponds a rearrangement of the conditionally convergent series $\sum_{n-1}^{\infty} C_{n}$ such that the rearranged series converges to $\beta$. In fact there are an infinity of such rearrangements with the sum $\beta$. So to any real number correspond an infinity of distinct points of $E$. Again to any other real number $\boldsymbol{\gamma}$, there correspond an infinity of other distinct points of $E$, each of which is different from any of those corresponding to $\beta$. Thus the set of real numbers is equivalent to a proper subset of the aggregate of points of $E$. Therefore $p \geqq C$. Combining these two, we have $p=c$.

THEOREM 3. Every point of the metric space $E$ is of degree $c$ (power of the continuum) in E.2

Proof. Take any point $x=\left(x_{1}, x_{2}, x_{3}, \cdots\right)$ of $E$. Then

$$
C\left(x_{1}\right)+C\left(x_{2}\right)+C\left(x_{3}\right)+\cdots
$$

is a rearrangement of the given conditionally convergent series. Let $N$ be a positive integer. Let $0<\alpha<1$ and let

2 The proof of Theorem 3 is due to the referee. It may be remarked that a proof of the above modelled on the plan of proof of Theorem 2 may also be easily given. 


$$
\alpha=. \alpha_{1} \alpha_{2} \alpha_{8} \alpha_{4} \cdots
$$

be the dyadic expansion of $\alpha$ which may terminate with zeros but not with ones.

Let $x_{n}^{(\alpha)}=x_{n}(n=1,2,3, \cdots, N+1)$ and if $\alpha_{p}=0$, let

$$
x_{N+2 p}^{(\alpha)}=x_{N+2 p}, \quad x_{N+2 p+1}^{(\alpha)}=x_{N+2 p+1}
$$

and if $\alpha_{p}=1$, let

$$
x_{N+2 p}^{(\alpha)}=x_{N+2 p+1}, \quad x_{N+2 p+1}^{(\alpha)}=x_{N+2 p .}
$$

Then to different numbers $\alpha$ correspond different points

$$
x^{(\alpha)}=\left(x_{1}^{(\alpha)}, x_{2}^{(\alpha)}, x_{8}^{(\alpha)}, \ldots\right) .
$$

All points $x^{(\alpha)}$ lie within the sphere with center $x$ and radius $2^{-N}$. And since $\lim C\left(x_{n}\right)=0$, the series

$$
C\left(x_{1}^{(\alpha)}\right)+C\left(x_{2}^{(\alpha)}\right)+C\left(x_{3}^{(\alpha)}\right)+\cdots
$$

has, for each $\alpha$, exactly the same limits of oscillation as the series $\sum C\left(x_{n}\right)$.

This proves that the points of $E$ that lie within the sphere $S\left(x, 2^{-N}\right)$ has the power of the continuum.

It proves more. It easily leads to the theorem:

ThEOREM 4. Every point of $E$ is the limit point of a set of points of $E$ of power $c$ at which the rearrangements of the conditionally convergent series behave in any prescribed manner.

Proof. Take any point $x=\left(x_{1}, x_{2}, x_{3}, \cdots\right)$. Let $l$ and $L$ be any two real numbers, $-\infty$ and $+\infty$ not excepted, such that $-\infty \leqq l \leqq L$ $\leqq+\infty$. In a sphere with $x$ as center and $2^{-N}$ as radius there exists a point $X=\left(x_{1}, x_{2}, \cdots, x_{N+1}, X_{N+2}, X_{N+3}, \cdots\right)$ at which the rearranged series has $l$ and $L$ as its limits of oscillation. Again, by the above, in a sufficiently small sphere with $X$ as center lying entirely within the first sphere, an infinite set of points of the power $C$ exist at which the rearranged series has the same limits of oscillation $l$ and $L$.

Thus in every neighbourhood of $x$, there exists a set of points of the power of the continuum, at which the rearranged series has any prescribed limits of oscillation.

DACCA, IndA 